\title{
DC-SQUID SENSOR SYSTEM FOR MULTICHANNEL NEUROMAGNETOMETRY
}

E.P. Houwman, D. Veldhuis, J. Flokstra, H.J.M. ter Brake, W. Jaszczuk, A. Martinez ${ }^{*}$ and H. Rogalla, University of Twente, Faculty of Applied Physics, P.O.Box 217, 7500 AE Enschede, The Netherlands, "Universidad de Zaragoza, E.T.S.I.I., Maria Zambrano 50, 50015 Zaragoza, Spain.

\section{Abstract}

Various DC-SQUID sensor configurations were developed for use in our 19-channel neuromagnetometer. Apart from the standard type, resistively and inductively shunted SQUIDs were made, allowing for a large screening factor $\beta(>1)$. In this way signal coupling from the pick-up coil to the SQUID is facilitated and capacitive coupling between the input coil and the SQUID washer can be decreased. The number of turns of the input coil is decreased further by allowing for an inductance mismatch in the input circuit. Although theoretically both measures give rise to an increased field noise of the sensor, they may lead to a reduction of the excess noise and the noise balance may become positive.

\section{Introduction}

DC-SQUIDs are currently the most sensitive detectors for measuring magnetic flux and are used in a wide variety of applications of which neuromagnetism is a very remarkable one. In this paper we describe the development of DC-SQUIDs, which will be used in a 19-channel DC-SQUID neuromagnetometer that is under construction at the University of Twente. The magnetometer channels consist of wire-wound, first-order gradiometer coils, connected to separately shielded SQUIDs. The field coils are placed in a hexagonal configuration over the curved bottom of the $125 \mathrm{~mm}$ diameter tail of a fibre-glass cryostat.

DC-SQUIDs are nowadays often fabricated as a washer type SQUID ring with a planar input coil on top ${ }^{2}$. For neuromagnetometers one often employs wire-wound field sensing coils, having inductances in the range $0.5-2 \mu \mathrm{H}$, which are connected to the planar input coil. Matching of this inductance with the input coil inductance, $L_{2}$, requires a large number of turns $\left(\mathrm{n}_{2}\right)$ of the planar input coil. $\mathrm{L}_{2}$ is approximately proportional to $\mathrm{n}_{2}^{2} \mathrm{~L}_{\mathrm{ln}}$, where $\mathrm{L}_{1 \mathrm{~h}}$ is the inductance of the hole in the SQUID washer. The planar input coil introduces a significant shunt capacitance across the SQUID loop, which increases with the number of turns of the input coil, and which forms an LC resonant circuit with the SQUID inductance ${ }^{3}$. Secondly, the input coil together with the SQUID washer as groundplane or the input coil as groundplane for the SQUID ring form stripline resonators ${ }^{4}$. The resonances can be excited by the high-frequency circulating currents in the SQUID ring and can give rise to a large amount of excess noise and thus to a performance degradation of the SQUID.

The aim of the study presented here, is to investigate the possibilities of reducing the capacitive coupling, maintaining the washer type SQUID configuration. A reduction of the coupling can of course be achieved by diminishing the layer overlap by narrowing the line width of the stripline. This is however limited by the technology available. Secondly one may allow for a mismatch between field sensing coil and input coil inductances. A third option is to increase the SQUID inductance, so that the number of turns of the input coil can be reduced to achieve the same input inductance. For the optimized standard type SQUID the screening parameter $\beta\left(=2 \mathrm{~L}_{1} \mathrm{I}_{0} / \Phi_{0}\right)$ is in the range from about 1 to 2 . Here $I_{0}$ is the critical current of a single Josephson junction and $L_{1}$ the SQUID inductance. For larger $\beta$ the voltage modulation depth decreases strongly. Thermal noise restricts $I$ to values larger than about $4 \mu \mathrm{A}$, for practical devices operated at LHe temperature. These restrictions limit $L_{1}$ to about 250 $500 \mathrm{pH}$ at the most. We investigated theoretically and experimentally two alternative devices, for which $\beta$ values larger than one are allowed, without a significant loss of voltage modulation depth ${ }^{6-8}$. This is achieved by the introduction of an inductive or resistive shunt across the SQUID loop. The price is some loss of sensitivity compared to the theoretical noise level of the optimized, standard type SQUID. However, in practice this loss may be compensated by a lower excess noise.
Standard as well as shunted washer type SQUIDs with a tightly coupled input coil were fabricated and characterized with respect to the noise and coupling properties.

The complete sensor is shortly described: the field sensing coil, the SQUID module, and the resonant output transformer.

\section{Theory}

The voltage modulation depth of the standard type SQUID in the optimum bias point depends on $\beta$ as $\Delta V_{m} \approx \Delta I_{I} R=I_{0} R /(1+\beta)$, where $R$ is the shunt resistance of the junction. $\Delta V_{m}$ decreases strongly with increasing $\beta$, thus limiting $\beta$ to values not much larger than 1 , for obtaining a not too small $\Delta V_{m}$ for SQUIDs with a small $\mathrm{I}_{0} \mathrm{R}$ product. The maximum flux-voltage transfer becomes $\partial V / \partial \Phi=\pi \mathrm{I}_{0} \mathrm{R} / \Phi_{0}(1+\beta)$, assuming a sinusoidal flux-voltage pattern. Optimum energy sensitivity is obtained for $\beta \approx 1$. The corresponding flux noise is (with $\gamma=9)^{5}$

$S_{\Phi}=\frac{\gamma}{4} \cdot \frac{2 \mathrm{k}_{\mathrm{B}} \mathrm{T} \Phi_{0}^{2}}{\mathrm{I}_{0}^{2} \mathrm{R}} \cdot \beta^{2}$.

In literature some multiloop SQUIDs have been described that combine a large coupling area with a small SQUID inductance by placing several inductances in parallel. The extreme case is a single large coupling loop attached to a small SQUID loop (figure 1a). It was shown that the I-V characteristics of the shunted SQUID are the same as those of a standard SQUID, when the effective parameters $\beta_{\mathrm{eff}} \approx\left(2 \mathrm{I}_{0} / \Phi_{0}\right)\left(\mathrm{L}_{\mathrm{sq}}+\mathrm{L}_{\mathrm{sh}}\right)$ and $\Phi_{\text {eff }}=\Phi /\left(1+\mathrm{L} / \mathrm{L}_{\mathrm{sh}}\right)$ are identified with $\beta$ and $\Phi$ of the standard SQUID, respectively ${ }^{6,10}$. Here, it is assumed that no flux is applied to the small loop and that $\mathrm{L}_{\mathrm{sh}} \ll \mathrm{L}$, as is the case in our experimental designs. $L_{s q}, L_{s h}$, and $L_{\text {are }}$ are inductances of the small loop, the inductive shunt, and the large loop, respectively. The voltage modulation depth can thus be approximated by $\Delta V_{\mathrm{m}} \approx \mathrm{I}_{0} R /\left(1+\beta_{\text {eff }}\right)$ The flux-voltage transfer becomes $\partial \mathrm{V} / \partial \Phi=\left(\pi \mathrm{I}_{0} \mathrm{R} / \Phi_{0}\left(1+\beta_{\mathrm{eff}}\right)\right) /\left(1+\mathrm{L} / \mathrm{L}_{\mathrm{sh}}\right)$. The voltage noise of the SQUID is approximately the same as that for the standard type SQUID, because there are no extra noise sources in the SOUID and most of the Josephson currents will pass through the shunt and not through the large loop. Therefore the flux noise spectral density of the applied flux is given by

$\mathrm{S}_{\Phi}=\frac{\gamma}{4} \cdot \frac{2 \mathrm{k}_{\mathrm{B}} \mathrm{T} \Phi_{0}^{2}}{\mathrm{I}_{0}^{2} \mathrm{R}} \cdot \beta^{2} \cdot\left(1+\mathrm{L} / \mathrm{L}_{\mathrm{sh}}\right)^{2}$,

where we assumed that the flux noise of the small loop with $\beta_{\text {eff }}$ is given by the numerical formula for the standard SQUID with $\beta \approx \beta_{\text {eff }} \approx 1$. The coupling loss from the large to the small loop is reflected in the term $\left(1+\mathrm{L} / \mathrm{L}_{\mathrm{st}}\right)^{-1}$ in $\partial \mathrm{V} / \partial \Phi$, and gives rise to the increased flux noise. This implies that for minimum noise in the large loop $\beta_{s h}$ must be made as large as possible, thus $\beta_{\text {efr }} \approx \beta_{\mathrm{sh}} \approx 1$, and $\beta_{\mathrm{sq}}^{\mathrm{sh}} \approx 0$. It is seen that $\Delta V_{\mathrm{m}}$ is practically independent of $\beta$, whereas $\left(S_{\Phi}\right)^{1 / 2}$ increases more or less linearly with $\beta$ as in the case of the standard SQUID.

A second way to have a small inductance loop for the Josephson currents and a large loop for the low frequency currents induced by the applied flux, is obtained by shunting the large loop with a resistive shunt, $R_{\text {sh }}$ (figure $\left.1 b\right)^{7,8}$. It was shown that $\Delta V_{\mathrm{m}} \approx I_{0} R / 2$ is nearly independent of $\beta$, if $\gamma=R / R_{\mathrm{sh}}$ is about 1 or larger, because the resistive shunt suppresses the $L C$ resonance of the SQUID strongly. The flux-voltage transfer is approximately equal to $2 \mathrm{~V} / \partial \Phi=\pi \mathrm{I}_{0} \mathrm{R} / 2 \Phi_{0}$. The flux noise spectral density was derived to be

$\mathrm{S}_{\Phi} \approx \frac{2 \mathrm{k}_{\mathrm{B}} \mathrm{T} \Phi_{0}^{2}}{\mathrm{I}_{0}^{2} \mathrm{R}} \cdot\left[\frac{4}{\pi^{2}}+\frac{\beta^{2}}{4}(1+2 \gamma)\right]$, 


\section{SQUID design and fabrication}
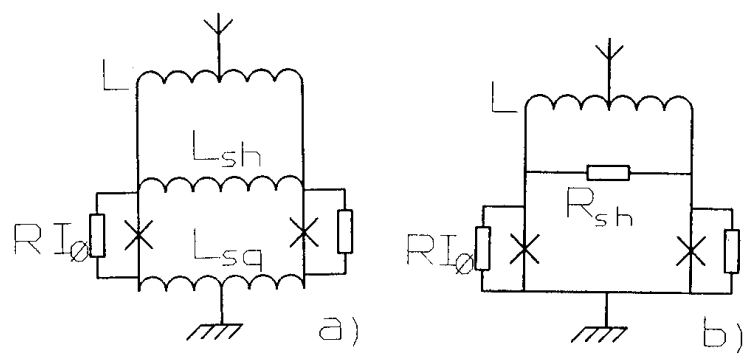

Figure 1: Inductively shunted SQUID (a) and resistively shunted SQUID (b).

for bias currents $I_{B}$ larger than the critical current $2 I_{0}$ of the SQUID. Here it is seen again that a large $\beta$ is on the expense of an increased flux noise. Further it was shown that these SQUIDs are hysteretic for $\mathrm{I}_{\mathrm{B}}<2 \mathrm{I}_{0}$, so that an increased flux noise may be expected for bias currents below $2 \mathrm{I}_{0}$.

The reduction of the number of turns of the input coil influences the mismatch between the inductances of the input and field sensing coils. The field-to-flux transfer function $F=\partial \Phi / \partial B$ of a SQUID magnetometer with a single flux-transformer input circuit is $F \approx A_{e f f} M_{12} /\left(L_{p}+L_{2}\right)$. Here, $B$ is the signal field in the field sensing coil with effective area $A_{\text {eff }}$ and inductance $L_{p}$ $M_{12}=k_{12}\left(L_{1} L_{2}\right)^{1 / 2}$ is the mutual inductance between input coil and SQUID and $\Phi$ is the signal flux in the SQUID. Using the ratio $\alpha=\mathrm{L}_{\mathrm{p}} / \mathrm{L}_{2}, \mathrm{~F}$ can be written as

$$
\mathrm{F} \approx \mathrm{L}_{1}^{1 / 2} \cdot \frac{\mathrm{A}_{\mathrm{eff}}}{\mathrm{L}_{\mathrm{p}}^{1 / 2}} \cdot \frac{\mathrm{k}_{12} \alpha^{1 / 2}}{(1+\alpha)}
$$

Here we assumed that the effective inductance of the input coil screened by the SQUID is approximately equal to the bare input inductance. Further it is assumed that the effective SQUID inductance, $L_{1, \text { eff }}=L_{1}\left(1-k_{12}^{2} /(1+\alpha)\right)$, is approximately equal to $L_{1}$, thus that $k_{12}<1$ and $\alpha>1$. The first term of eq.[4] is determined by the SQUID, the second by the field sensing coil used and the third term describes the coupling. The field sensitivity $B_{n}$ of the magnetometer can now straightforwardly be estimated from the flux noise of the SQUID

$B_{n} \approx \frac{S_{\Phi}^{1 / 2}}{F}=\frac{S_{\Phi}^{1 / 2}}{L_{1}^{1 / 2}} \cdot \frac{1+\alpha}{\alpha^{1 / 2}} \cdot \frac{L_{p}^{1 / 2}}{A_{e f f}}$.

The first term is proportional to the root of the SQUID energy sensitivity and is rougly proportional with $\beta^{1 / 2}$ for $\beta>1$ for all SQUIDs discussed. $B_{\mathrm{n}}$ increases only slowly with increasing $\alpha$. So is $B_{n}(\alpha=10)$ only 1.7 times as large as $B_{n}(\alpha=1)$. The loss of sensitivity may be compensated by a lower $S_{\Phi}$, because of less excess noise due to resonances. Further coupling to a highinductance field coil is achieved more easily. The capacitive coupling between SQUID and input coil is largely determined by the number of turns $n_{2}$, which is easily obtained as

$\mathrm{n}_{2} \approx\left(\mathrm{L}_{\mathrm{p}} / \alpha \mathrm{L}_{1}\right)^{1 / 2}=\left(2 \mathrm{~L}_{\mathrm{p}} \mathrm{I}_{0} / \alpha \beta \Phi_{0}\right)^{1 / 2}$.

Thus $n_{2}$ can be decreased considerably if $\alpha$ is increased from 1 to 10 . Secondly, a large $\beta$ SQUID also leads to a decrease of $n_{2}$ It is noted that the outer turns of a planar input coil contribute more to the capacitive coupling than the inner turns, whereas an increased size of the SQUID hole leads to an increased capacitive coupling per turn because of the increased length of a winding. Thus the capacitive capacitive is reduced more by increasing $\alpha$ than by a larger $\beta$.
A process for the fabrication of all $\mathrm{Nb}$ DC-SQUIDs was developed on the basis of $\mathrm{Nb} / \mathrm{Al}, \mathrm{AlO}_{\mathrm{x}}(, \mathrm{Al}) / \mathrm{Nb}$ technology ${ }^{11}$. The junction areas are defined by a standard SNAP process and are characterized by gap voltages of typically $2.8-2.9 \mathrm{mV}, \mathrm{I}_{0} \mathrm{R}$ products of $1.7-1.9 \mathrm{mV}$, i.e. $70-80 \%$ of the theoretical value and $V_{m}$ values of up to $70-80 \mathrm{mV}$ at $4.2 \mathrm{~K}$ and over $1 \mathrm{~V}$ at $1.6 \mathrm{~K}$. In the SQUID the junctions, with critical currents of 5-15 $\mu \mathrm{A}$, are shunted by thin film Pd resistors to obtain $\beta_{\mathrm{c}}$ values of $0.1-0.5$. All structuring was done by lift-off, using a chlorobenzene process step and standard $5 \mu \mathrm{m}$ photolithography. The SQUIDs consist of a $\mathrm{Nb} / \mathrm{Al}, \mathrm{AlO}_{\mathrm{x}} / \mathrm{Nb}$ trilayer of which the top lay$\mathrm{er}$ is anodized in order to define the junction areas. For the insulation between the input coil and the SQUID washer and parts of the structure that are not anodized, we used a double $\mathrm{SiO}_{2}$ layer. The first layer of $250 \mathrm{~nm}$ thickness is used to fill the spaces between the deposited structures. The second layer with a thickness of $150 \mathrm{~nm}$ is RF sputtered, with $-75 \mathrm{~V}$ voltage bias on the substrate in order to obtain edge rounding and thus smoothly indulating surfaces.

In table 1 the design parameters of the different SQUIDs are given.

\begin{tabular}{|c|c|c|c|c|c|c|c|}
\hline \multirow[b]{2}{*}{ SQUID } & \multicolumn{4}{|c|}{ design parameters } & \multicolumn{3}{|c|}{ experimental results } \\
\hline & $\mathrm{L}_{1 \mathrm{~h}}(\mathrm{pH})$ & $\mathrm{n}_{2}$ & $\mathrm{~L}_{2}(\mu \mathrm{H})$ & $M_{12}(\mathrm{nH})$ & $\mathrm{L}_{1}(\mathrm{pH})$ & $M_{12}(n H)$ & $k_{12}$ \\
\hline Sta1 & 110 & 28.5 & 0.095 & 3.1 & 150 & 2.2 & 0.58 \\
\hline Sta2 & 230 & 20.5 & 0.10 & 4.7 & 270 & 4.1 & 0.79 \\
\hline Res1 & 240 & 21 & 0.11 & 5.0 & 385 & 5.2 & 0.80 \\
\hline Res2 & 300 & 21 & 0.21 & 9.9 & 670 & & 0.79 \\
\hline $\operatorname{Res} 3$ & 1330 & 13 & 0.22 & 17.3 & 1480 & & \\
\hline Ind 1 & 110 & 21 & 0.21 & 0.29 & 160 & 0.29 & 0.050 \\
\hline Ind2 & $110^{\circ}$ & 10 & 0.32 & 0.18 & 160 & 0.17 & 0.024 \\
\hline
\end{tabular}

Inductance of small hole; large hole inductance $L_{1 h}$, is 470 and $3100 \mathrm{pH}$ for Ind 1 and Ind2, respectively.

The junctions of the standard type SQUIDs are placed at the inside of the washer to avoid the parasitic slit inductance. In case the junctions are placed at the outside of the washer there is the return line of the input coil in the slit in the washer. The associated inductance is estimated with a formula for a double slot line to be about $0.34 \mathrm{pH} / \mu \mathrm{m}$. We avoided as much layer overlap as possible. Therefore the junction structure is relatively open, as can be seen from fig. 2 , showing the central region of a typical standard type SQUID.

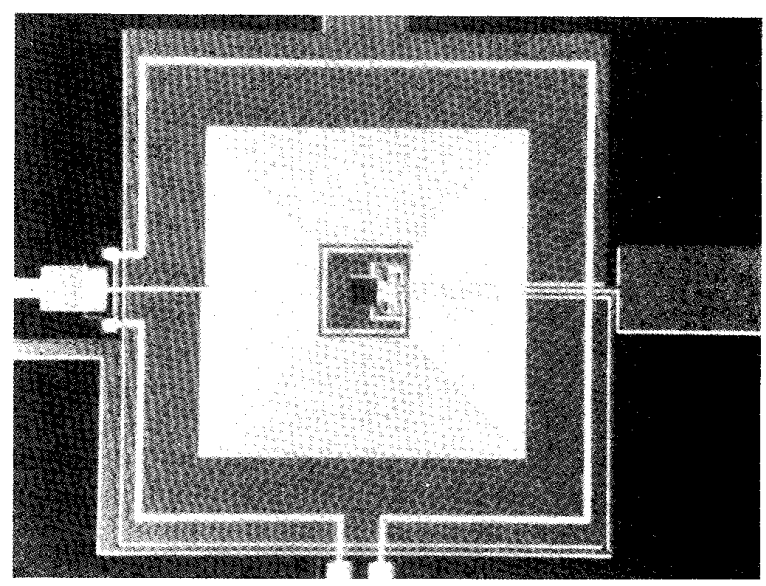

Figure 2: Standard DC-SQUID. Hole size is $70 \mu \mathrm{m}$, corresponding to an hole inductance of $110 \mathrm{pH}$. 
In the case of the resistively shunted SQUIDs the junctions and the shunt resistor $R_{s h}$ are placed at the outside of the washer, because the slit inductance, $L_{\text {slit, }}$ becomes relatively small compared to the hole inductance.

The inductively shunted SQUIDs consist of a small washer with the junctions at the inside and with a hole inductance of $110 \mathrm{pH}$, on which the modulation coil is placed. This washer is attached to a large washer with hole inductance $\mathrm{L}_{1 \mathrm{~h}}$. The input coil is placed around the large hole. The shunt inductance, $\mathrm{L}_{\mathrm{sh}}$, is estimated from the ratio of the length of the superconducting shunt to the circumference of the small loop to be $18 \mathrm{pH}$. This value is much smaller than the hole inductance, thus one may expect a fairly poor coupling from the input coil to the small loop. The corresponding mutual inductance is estimated as $\mathrm{M}_{1 \cdot 2} \mathrm{~L}_{\mathrm{sh}} /\left(\mathrm{L}_{\mathrm{lh}}+\mathrm{L}_{\mathrm{slit}}+\mathrm{L}_{\mathrm{sh}}\right)$, to be 0.29 and $0.18 \mathrm{nH}$, respectively. Here, $M_{1} \approx n_{2} L_{1 h}$, is the mutual inductance between the input coil and the large hole.

\section{Results and discussion}

The I-V and V- $\Phi$ characteristics of all the SQUIDs produced are generally smooth in the normal bias range up to about (3-4) $I_{0}$, where the first crossover in the I-V curves for $\Phi=n \Phi_{0}$ and $(n+1 / 2) \Phi_{0}$ is present. We can explain this crossover by the well-known LC resonance in the SQUID. At higher voltages more crossovers are present, indicating resonances at higher frequencies. In the case of the resistively shunted SQUTDs the first LC resonant step is fully suppressed by the shunt resistor, so that a large voltage modulation depth is possible, even in the case of large- $\beta$ SQUIDs.

In figure 3 the experimental values for the normalized voltage modulation depth at a bias current of $2.1 \mathrm{I}_{0}$ for the different types of SQUIDs are shown as function of the screening parameter $\beta$. The data for the standard type SQUID decrease strongly with increasing $\beta$ and are fairly well described by $\Delta V_{m} / I_{0} R=(1+\beta)^{-9}$. The $\Delta V_{m} / I_{0} R$ values for the inductively and resistively shunted SQUIDs are mostly slightly larger than 0.5 and practically independent of $\beta$. The scatter of the values of the inductively shunted SQUIDs is due to the fact that $\beta_{\text {eff }}$ is not exactly equal to 1 for all the SQUIDs. It was observed that the resistively shunted SQUIDs with the large loops are very sensitive for extemal noise (probably RF interference), that often wipes out part or even most of the voltage modulation. This is probably the reason for the scatter of the experimental $\Delta \mathrm{V}_{\mathrm{m}} \mathrm{val}$ ues of these SQUIDs.

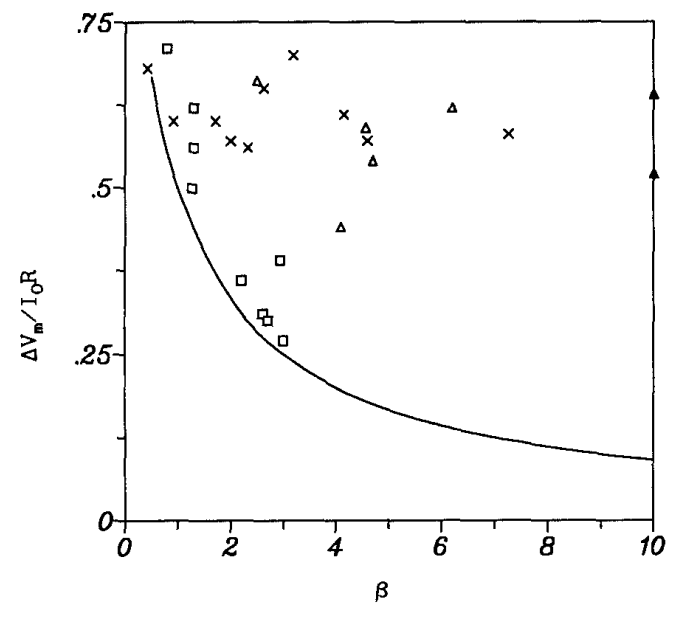

Figure 3: Normalized voltage modulation depth of standard. Standard SQUID: $\Delta \mathrm{V}_{\mathrm{m}} / \mathrm{I}_{0} \mathrm{R}=1 /(1+\beta)$ denoted by —; exp. data: $\square$. Resistively shunted SQUID: $\mathbf{x}$. Inductively shunted SQUID: $\Delta$.
The inductances of the standard and inductively shunted SQUIDs, which have $\beta$, respectively $\beta_{\text {eff }}$ about equal to 1 , were determined from the critical current modulation depth $\Delta \mathrm{I}_{\mathrm{c}}$. The values obtained, given in table 1 , are about $40-50 \mathrm{pH}$ larger than the inductances calculated from the hole size. For these SQUIDs $\mathrm{L}_{1}$ is made up of $\mathrm{L}_{1 \mathrm{~h}}$ and the inductance $\mathrm{L}_{\mathrm{J}}$ associated with the junction structure. Therefore we estimated $L_{J}$ to be in the range $40-50 \mathrm{pH}$. This relatively large value is probably mostly due to the 'open' junction structure: the area enclosed by the shunt resistors and the junction is a significant fraction of the hole area. It was expected that for the resistively shunted SQUIDs one can not determine $L_{1}$ from $\Delta I_{c}$ because of the hysteresis at $\mathrm{I}_{B}<2 \mathrm{I}_{0}$. However we did not observe any hysteresis and the critical current modulation depth is fairly well given by $\Delta \mathrm{I}_{\mathrm{c}} \approx 2 \mathrm{I}_{0} /(1+\beta)$. The error made in the determination of $\mathrm{L}_{1}$ from $\Delta \mathrm{I}_{\mathrm{c}}$ is fairly large for these large $\beta$ SQUIDs. Therefore, $L_{1}$ is calculated as the sum of the estimated hole, junction, and slit inductances.

The mutual inductances between the SQUID and the input coil, $M_{12}$, determined from the current needed to produce one $\Phi$ in the SQUID, as well as the coupling factors $k_{12}=M_{12}\left(L_{1} L_{2}\right)^{-1 / 2}$. calculated from the $L_{1}$ and $L_{2}$ values of table 1 , are given in table 1 also. The fairly poor coupling, $k_{12} \approx 0.58$, for the standard type SQUID Sta1 is ascribed to the relatively large contribution of $\mathrm{L}_{\mathrm{J}}$ to $\mathrm{L}_{1}$ (this accounts for approximately $15 \%$ of the coupling loss) and the flux leakage through the open junction structure and slit in the washer, that is no part of the coupling area. For the SQUID Sta2 the relative effect of the slit and $L_{J}$ is about twice as small as for the SQUID Stal. Therefore the coupling loss is also reduced with about a factor of 2 . The measured mutual inductances of the inductively shunted SQUIDs are nearly equal to our estimates. This indicates that the coupling between the input coil and the large loop is characterized by a coupling factor that is nearly equal to 1 and that most of the coupling loss can be accounted for by the poor coupling to the small loop. The $\mathrm{M}_{12}$ of the resistively shunted SQUID Res1 is even slightly larger than the predicted value. This is probably because part of the slit contributes to the coupling area. The flux leakage and the relatively small $L_{J}$ compared to $L_{1 h}$, result in a $k_{12}$ value smaller than 1 .

Table 2: Measured $f$ lux and fleld nolse levels
\begin{tabular}{|l|llllll|}
\hline SQUID & $\Phi_{\mathrm{n} . t h}$ & $\Phi_{\mathrm{n} . \exp }\left(\mu \Phi_{0} / \sqrt{\mathrm{Hz}}\right)$ & $\mathrm{B}_{\mathrm{n}}(\mathrm{fT} / \sqrt{\mathrm{Hz}})$ & $\alpha$ & $\beta$ \\
\hline Sta1-1 & 1.2 & 3.8 & 3.5 & 9.3 & 1.4 \\
Sta1-2 & 1.2 & 8.4 & 7.8 & 9.3 & 1.5 \\
Sta2-1 & 2.1 & 10 & 5.0 & 8.6 & 3 \\
Sta2-2 & 1.9 & 15 & 7.5 & 8.6 & 2 \\
Res2 & 1.3 & 2.6 & 1.7 & 4.0 & 2.4 \\
Res3 & 7.9 & 23 & 3.6 & 3.8 & 9.3 \\
Ind1 & 1.6 & 3.5 & 28 & 4.0 & 1.1 \\
Ind2 & 1.2 & 1.8 & 27 & 2.6 & 1.6 \\
\hline
\end{tabular}

With shorted input coll: measured $\mathrm{L}_{1}=230 \mathrm{pH}$.

The flux noise spectral densities of various SQUIDs with open input coil were measured in a standard $100 \mathrm{kHz}$ Flux Locked Loop with square wave flux modulation. In table 2 the minimum experimental flux noise values, $\Phi_{\text {nexp }}$, are given, as well as the theoretical values, $\Phi_{\text {nth }}$. In the case of the inductively shunted SQUIDs one measures the intrinsic flux noise of the small loop. The noise in the large loop coupled to the input coil, is obtained by multiplication with the factor $\left(\mathrm{L}_{\mathrm{sit}}+\mathrm{L}_{\mathrm{sh}},+\mathrm{L}_{\mathrm{sh}}\right) / \mathrm{L}_{\mathrm{sh}}$. The correspondence between the experimental and theoretical flux noise spectral densities for these SQUIDs are considered to be good. For the standard type SQUIDs we find a flux noise that is increased by at least a factor of 3. This excess noise is ascribed to resonances in the SQUID, due to the tight coupling to the input coil, in contrast with the inductively shunted SQUIDs in which the high frequency circulating currents are shunted away from the large loop by the shunt inductance. The inductively shunted and standard SQUIDs show a 
fairly shallow minimum in the flux noise at $\mathrm{I}_{\mathrm{B}}=1.7-1.8 \mathrm{I}_{0}$, as expected. The flux noise of the resistively shunted SQUID is a factor 2 to 3 larger than the calculated values. There is hardly any dependence on the bias current. The small excess noise is probably due to resonances introduced by the coupling to the input coil. However, these resonances and the high-frequency circulating currents in the loop are damped by the shunting resistor $\mathrm{R}_{\mathrm{sh}}$, contrary to the standard SQUID. This may be the reason for the lower excess noise. In all cases the low frequency noise starts below about $1 \mathrm{~Hz}$, but the frequency dependence is not $1 / \mathrm{f}$ and differs from SQUID to SQUID. Therefore we think that this noise is dominated by some external source.

\section{Sensing coil and SQUID module}

The field sensing coil is a wire-wound, first-order gradiometer, having two sections of 3 turns each, with a diameter of $20 \mathrm{~mm}$ and a baseline length of $40 \mathrm{~mm}$. The distance between the turns of the field sensing section is $0.5 \mathrm{~mm}$, whereas that of the turns of the compensating section is $6 \mathrm{~mm}$. The effective area of the sensing coil is about $9.46 \mathrm{~cm}^{2}$ and the self-inductance of the coil plus connecting leads is $0.83 \mu \mathrm{H}$. The SQUID chip is placed in a rigid, compartmentalized $\mathrm{Nb}$ module, consisting of several shielded sections. A small resonant transformer is used between the SQUID and the preamplifier. The selected gain of the transformer is 40 . With a moderate Q-factor of 10 the transformer voltage gain must be about equal to 4 . A prototype transformer of $0.1 \mathrm{~mm} \mathrm{Cu}$ wire, with a length of $5 \mathrm{~mm}$ and an outer diameter of $5.8 \mathrm{~mm}$, and consisting of 90 primary and 635 secundary turns, was made. The measured inductances are $17.4 \mu \mathrm{H}(27.5 \mu \mathrm{H})$ and $490 \mu \mathrm{H}(760 \mu \mathrm{H})$ respectively. The coupling factor appeared to be $0.81(0.89)$, giving a voltage gain of $4.3(4.7)$. The values between brackets were measured outside the superconducting enclosure. The series resistances of the coils are well below $1 \Omega$, thus the noise contribution is negligible. A shunt capacitor across the secondary coil, placed inside the module, is used to bring the transformer into resonance.

\section{Field sensitivity}

With regard to the considered application we calculated the expected white field noise in the sensing coil on the basis of the measured flux noise levels and the described gradiometer coil. The effect of screening of the SQUID inductance by the input circuit on the flux noise is realtively small and is therefore neglected, because in all cases $k_{12}<0.8$ and $\alpha>3$. The field sensitivity is estimated as $B_{n} \approx \Phi_{0}$ exp $\left(L_{p u}+L_{2}\right) / M_{1} A_{\text {. }}$. The results given in table 2 , show that $B_{n}$ is less than $8 \mathrm{fT} / \mathrm{Hz}$ for the resistively and standard SOUIDs, reaching a minimum of $1.7 \mathrm{fT} / \mathrm{VHz}$ for the smallest resistively shunted SQUID measured. The increased field noise of the inductively shunted SOUIDs is due to the poor coupling from the large to the small loop. The shunt inductance can probably be increased to about $90 \mathrm{pH}$. Leaving the structure further unaltered, the coupling increases significantly and the field noise would become as low as about $6 \mathrm{fT} / \mathrm{VHz}$ for these SQUIDs. When the SQUIDs are used with a superconducting pick-up coil the field noise of the SQUID sensors may be increased because of resonances in the SQUID system due to coupling to the input circuit. However it is expected that, in the case of the shunted SQUIDs the extra noise is much less than for the standard SQUIDs, because the high-frequency circulating currents are shunted away from the input circuit.

\section{Conclusions}

Various DC-SQUID sensor configurations were developed for use in our 19-channel neuromagnetometer, presently under construction. A controllable and reliable process, based on $\mathrm{Nb} / \mathrm{Al}$ technology, leads to SQUIDs with well-behaved I-V and V- $\Phi$ characteristics. Resistively and inductively shunted SQUIDs allow large $\beta$ values, enabling a reduction of the number of turns of the input coil. This number is reduced further by allowing for an inductance mismatch in the input circuit. The capacitive coupling between input coil and SQUID is thus reduced probably leading to smaller excess noise. Although theoretically the noise increases with increasing $\beta$ and mismatch factor $\alpha$ the noise balance may be positive.

\section{References}

(1) H.J.M ter Brake et al., "Design and construction of a 19channel DC-SQUID neuromagnetometer", Physica B165\&166, 95, 1990.

(2) F. Dettman et al., "A monolithic thin film DC-SQUID", Phys. Stat. Sol. (a) 51, K185, 1979;

J.M. Jaycox and M.B. Ketchen, "Planar coupling scheme for ultra low noise DC-SQUIDs", IEEE Trans.Magn. MAG-17, 400,1981;

M.B.Ketchen,"DC-SQUIDs 1980: the state of the art",idem,387.

(3) C.D. Tesche, "Analysis of a double-loop DC-SQUID", J. Low Temp. Phys. 47, 385, 1982.

(4) B. Muhlfelder et al., "Well coupled, low noise, DC SQUIDs", IEEE Trans. Magn. MAG-21, 427, 1985.

(5) C.D. Tesche and J. Clarke, "DC-SQUID: noise and optimization", J. Low Temp. Phys. 29, 301, 1977;

J.J.P. Bruines et al., "Comment on: 'DC-SQUID: noise and optimization' by Tesche and Clarke", J.Low Temp.Phys. 46,383, 1982. (6) H. Koch, "An inductively shunted SQUID", Proc. ISEC I0, 384, 1984.

(7) H. Koch, "DC-SQUID design for a 25-channel multisensor test circuit", SQUID'85, 1079, 1985.

(8) K. Enpuku et al., "Effect of damping resistance on voltage versus flux relation of a DC-SQUID with large inductance and critical current, J. Appl. Phys., 1691, 1985.

(9) V.J.de Waal and T.M.Klapwijk, 'Compact integrated DC-SQUID gradiometer", Appl. Phys. Lett. 41, 669, 1982;

M.W. Cromar and P. Carelli, 'Low noise tunnel junction DCSQUIDs", Appl. Phys. Lett. 38, 723, 1981.

(10) E.P. Houwman, thesis University of Twente, Enschede, The Netherlands, 1990

E.P.Houwman et al., "Comparison of shunted DC-SQUIDs with large B", Physica B165\&166, 81, 1990.

(11) E.P. Houwman et al., "Fabrication and properties of $\mathrm{Nb} / \mathrm{Al}, \mathrm{Alox} / \mathrm{Nb}$ Josephson tunnel junctions with a double oxide barrier", J. Appl. Phys. 67, 1992, 1990;

D.J. Adelerhof et al., "Conductance studies on different types of $\mathrm{Nb} / \mathrm{Al}, \mathrm{Alox}(/ \mathrm{Al}) / \mathrm{Nb}$ Josephson tunnel junctions", Physica B165\&166, 1581, 1990;

D.J. Adelerhof et al., "Characterization of different types of $\mathrm{Nb}-\mathrm{AlO}_{\mathrm{x}}$ based Josephson tunnel junctions", these proceedings. 